\title{
Supporting Information \\ Quantum chemical prediction of electron ionization mass spectra of trimethylsilylated metabolites
}

Shunyang Wang ${ }^{(1)(2)}$, Tobias Kind ${ }^{(1)}$, Parker Ladd Bremer ${ }^{(1)}$, Dean J. Tantillo ${ }^{(2)}$ and Oliver Fiehn ${ }^{(1)^{*}}$

(1) West Coast Metabolomics Center, UC Davis Genome Center, University of California, 451 Health Sciences Drive, Davis, California 95616, United States

(2) Department of Chemistry, University of California, 1 Shields Ave, Davis, California 95616, United States

${ }^{(*)}$ Corresponding author Email

ofiehn@ucdavis.edu

Table of Content

Supplemental Scheme

P. 2

Supplemental Tables

P. 2-3

Supplemental Trajectory analysis

P. 3-4

Supplemental Mass spectral fragmentation rules (Part II)

P. 4-8

Supplemental accurate mass spectra

P. 9

Estimate Processing time of QCEIMS TMS compounds

P. 9-10

Supplemental Figure s12

P.10

Supplemental Reference

P. 10-11 


\section{Scheme S1. Flowchart of compound classification}

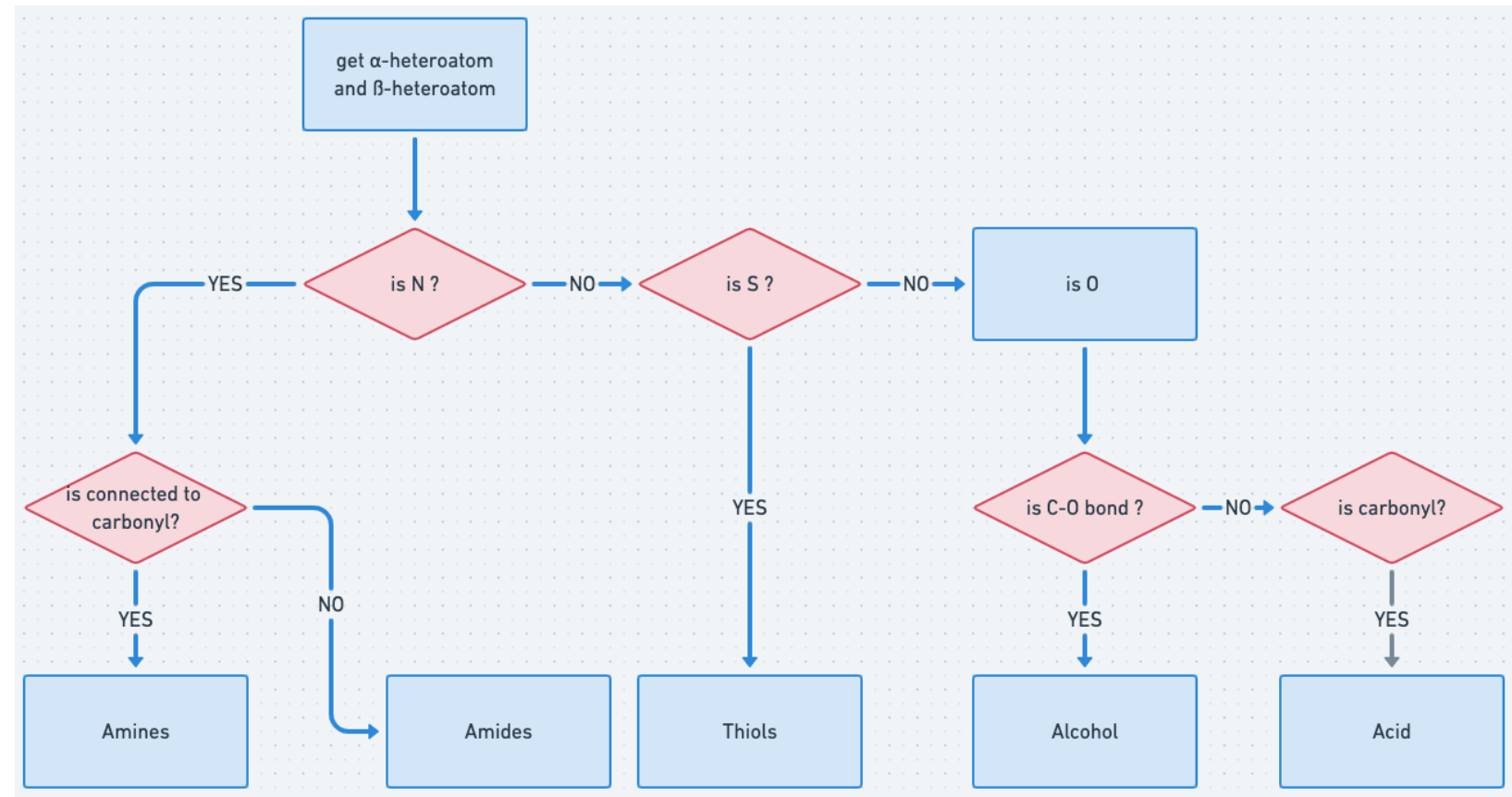

Table S1. Mass spectral similarities of selected compounds in different classes and their cosine and dot-product similarity scores when compared against experimentally obtained reference mass spectra.

\begin{tabular}{cccc}
\hline$\#$ & Name & Dot & Cos \\
\hline 305 & trimethylsilyl 2-amino-4-methylpentanoate & 373 & 697 \\
$470^{\text {a }}$ & trimethylsilyl 4-methoxybenzoate & 729 & 575 \\
$486^{\text {a }}$ & 2-(2-methoxyphenyl) ethoxy-trimethylsilane & 571 & 585 \\
491 & trimethyl-(2-propoxyphenoxy) silane & 724 & 529 \\
587 & 5-pyridin-3-yl-1-trimethylsilylpyrrolidin-2- & 517 & 473 \\
$\quad$ one & & \\
566 & 5-methyl-1-trimethylsilylindole-2,3-dione & 363 & 596 \\
444 & 1-phenyl-2-(trimethylsilylamino) propan-1- & 665 & 665 \\
& one & \\
535 & 1-N-trimethylsilylnaphthalene-1,5-diamine & 898 & 538 \\
501 & trimethyl(2-phenoxyethylsulfanyl) silane & 47 & 454 \\
648 & methyl 2-trimethylsilylsulfanylbenzoate & 840 & 695 \\
\hline
\end{tabular}

a. Discussed in the paper

Table S2. Dot and Cos similarity scores of multi-TMS-derivative compounds 


\begin{tabular}{cccccc}
\hline NAME & TMS & \#ATOM & ExactMass & Dot & Cos \\
\hline Methylamine, 2TMS derivative & 2 & 31 & 175.1212 & 712 & 458 \\
Hydroxylamine, 2TMS derivative & 2 & 29 & 177.1005 & 746 & 483 \\
Formamide, 2TMS derivative & 2 & 30 & 189.1005 & 633 & 428 \\
Ethanamine, 2TMS derivative & 2 & 34 & 189.1369 & 589 & 513 \\
Methoxyamine, 2TMS derivative & 2 & 32 & 191.1161 & 524 & 321 \\
Trimethylsilylpropargyl alcohol, 2TMS & 2 & 32 & 200.1052 & 327 & 136 \\
derivative & 2 & 35 & 201.1369 & 674 & 440 \\
Allylamine, 2TMS derivative & 2 & 37 & 203.1525 & 438 & 299 \\
Propylamine, 2TMS derivative & 2 & 32 & 204.1001 & 890 & 777 \\
1,2-Ethenediol, 2TMS derivative & 3 & 41 & 249.1400 & 159 & 336 \\
Hydroxylamine, 3TMS derivative & 3 & 44 & 276.1509 & 575 & 151 \\
Urea, 3TMS derivative & 3 & 47 & 277.1713 & 300 & 348 \\
Ethanolamine, 3TMS derivative & 3 & 45 & 284.1560 & 660 & 515 \\
3,4-Bis(trimethylsilyl)-1H-pyrazole, & 3 & & & & \\
3TMS derivative & 3 & 46 & 291.15060 & 550 & 290 \\
\hline Glycine, 3TMS derivative & & & & &
\end{tabular}

\section{Trajectory analysis}

For example (Figure S1, \#305, L-Leucine-TMS derivative), the molecular ion peak in the reference spectrum almost disappeared and in the in-silico spectrum it is relatively high abundant. The Figure S1b shows the fragments generated by MassFrontier and validated by our accurate in-silico mass spectrum. It is a common situation that under $70 \mathrm{eV}$ most of the molecular ions are fragmented and result in a low abundant molecular ion peak, while the $[\mathrm{M}-117]^{+}$peak (loss of COOTMS ${ }^{\bullet}$ ) becomes the base peak. Thus, the molecular ion has a shorter lifetime in the experiment, and we didn't provide enough energy to break the molecules in the simulation.

The figure S1c scans the bond length between the $\mathrm{CH} 3$ and the Si under high level calculation (pbe0/6-31G(d)) on a more general model. It shows that a four-member ring generation after the loss of ${ }^{\bullet} \mathrm{CH} 3$, which is also validated by the QCEIMS simulation. 


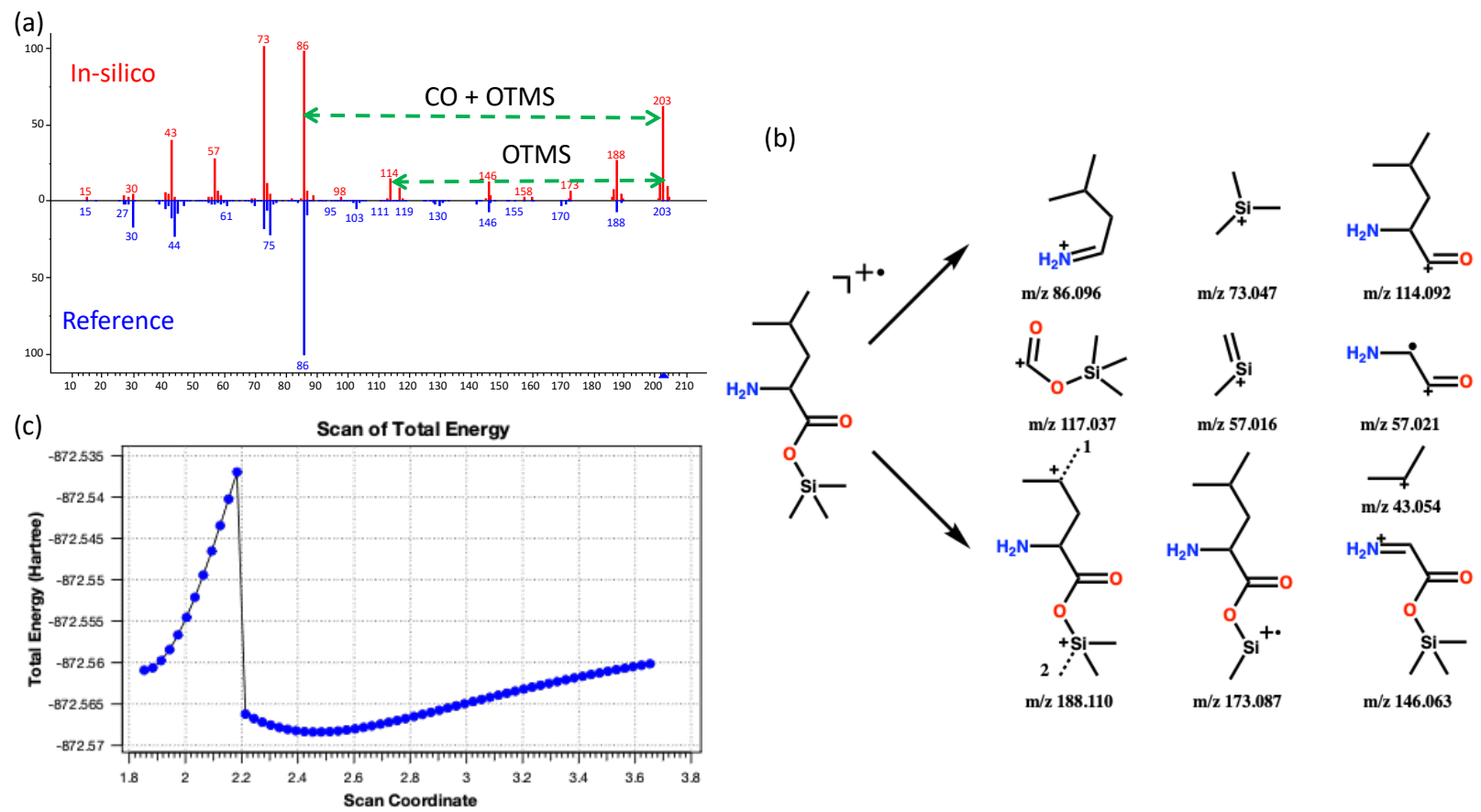

Figure S1. Fragmentation analysis of carboxylic groups; (a) head-to-tail spectrum of trimethylsilyl leucinate against reference spectrum; (b) fragments found in the simulation; (c) energy change of bond length scan

(In-silico spectrum available at https://mona.fiehnlab.ucdavis.edu/spectra/display/MoNA040855)

\section{Mass spectral fragmentation rules (Part II)}

\section{Alcohols}

We discussed two alcohol isomers with different aromaticity. Figure S2 shows an aromatic alcohol compound. The $[\mathrm{M}-\mathrm{CH} 3]^{+}$and $m / z 73$ peak are characteristic peaks of TMS derivatives. The fragmentations of $\mathrm{m} / \mathrm{z} 194$ and $\mathrm{m} / z 120$ peak are found in the simulation and validated by the MassFrontier prediction, but those peaks are not in the experimental spectrum.

One interesting observation is peaks $\mathrm{m} / \mathrm{z} 135$ and $\mathrm{m} / \mathrm{z} 136$ (Figure S2c). There are 18 trajectories generating $m / z 135$ and one peak generating the peak $m / z$ 136. Whether the hydrogen on position $\mathrm{B}$ migrates to position A decides the different positive charge sites of peak $\mathrm{m} / \mathrm{z} 135$. Rather than the Si-O-O-C four-member ring structure proposed by MassFrontier, the simulation only found a radical ion $\mathrm{m} / \mathrm{z} 136$ peak. But in this trajectory (TMP.642), a sequential hydrogen atom rearrangement is observed. The five-member ring in $\mathrm{m} / \mathrm{z} 136$ and 151 is also a typical structure of ortho-oxygen aromatic compounds. ${ }^{1}$ 

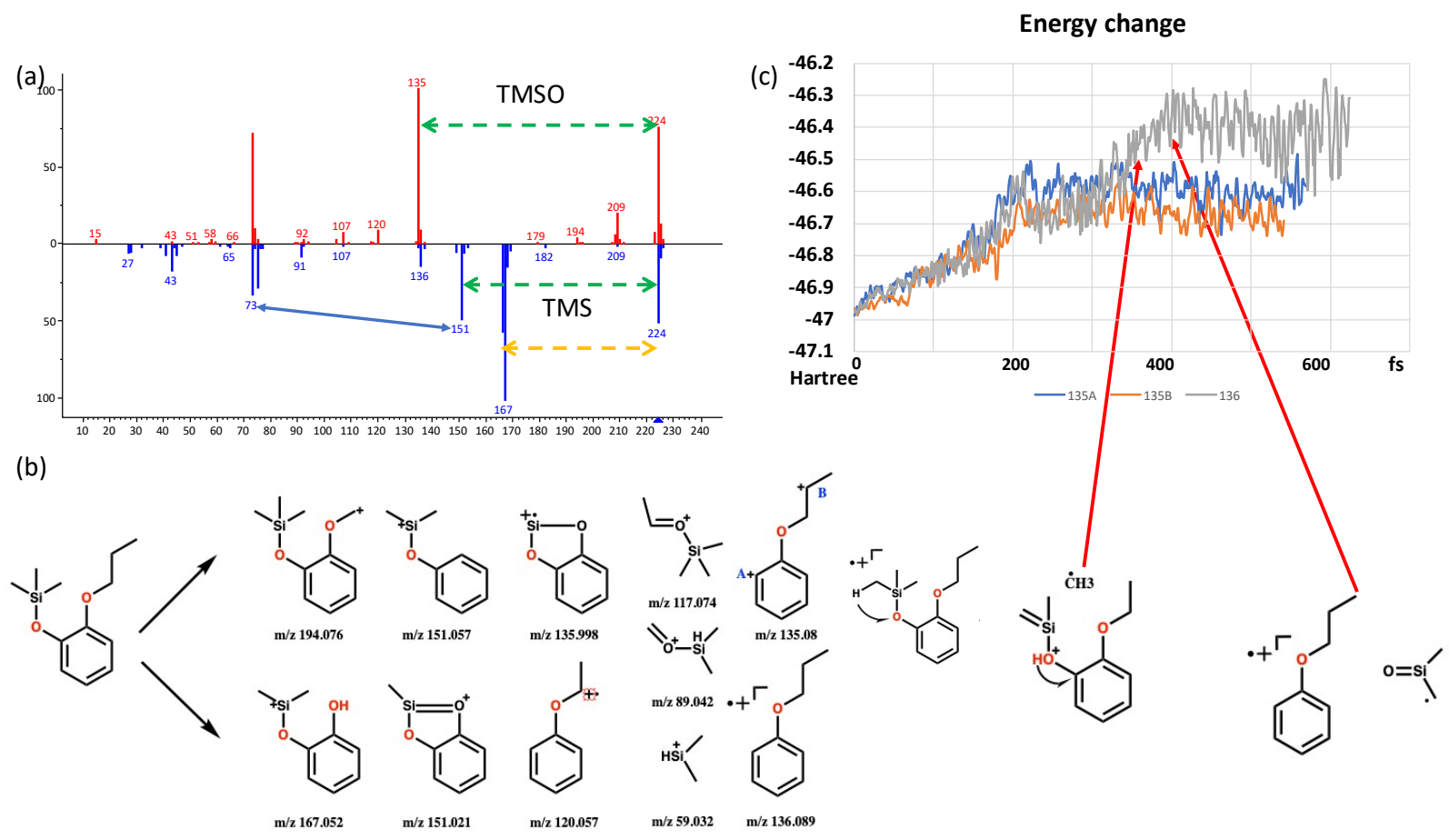

Figure S2. Fragmentation analysis of aromatic carboxylic group; (a) head-to-tail spectrum of 2propoxyphenol, TMS derivative against reference spectrum; (b) fragments found in the simulation; (c) energy change $m / z$ peak 135 and 136

(In-silico spectrum available at https://mona.fiehnlab.ucdavis.edu/spectra/display/MoNA040498)

\section{Amides}

Amides contain amine groups next to a ketone functional group. In the case which nitrogen is adjacent to an aromatic carbon, we assigned the molecules as aromatic amides. Amides have a similar structure to carboxylic acids and similar fragments in the mass spectrum are observed. ${ }^{2-3}$ For Aliphatic amides (\#587, Figure S3), the simulation reproduced the dominant peaks $(\mathrm{m} / \mathrm{z} 234$, $219,165,145,73$ ) quite well, but missed the $\mathrm{m} / \mathrm{z} 118$ peak, which is only one hydrogen less than the peak at $m / z 119$. The generation of $\mathrm{C} 8 \mathrm{H} 8 \mathrm{~N}^{+}$peak contains a nitrogen rearrangement and is shown in the simulation energy plots. The fragmentation reaction started with a TMS group migrating from nitrogen to oxygen (compound A). From compound $\mathrm{B}$ to compound $\mathrm{C}$, the energy kept increasing, while it is equilibrated from $\mathrm{C}$ to $\mathrm{D}$. We can find four main structures from the trajectory 432 . 


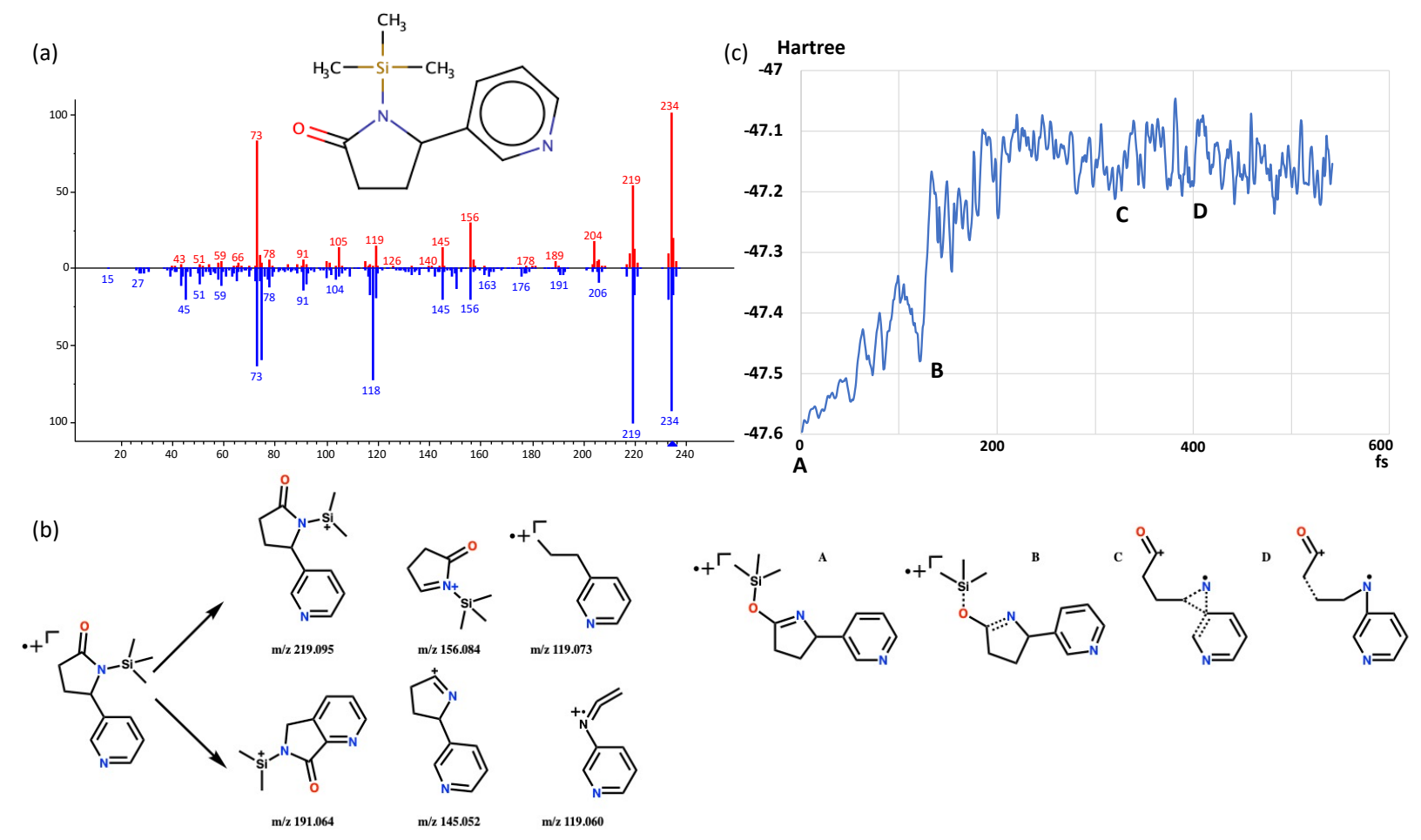

Figure S3. Fragmentation analysis of aromatic carboxylic group; (a) head-to-tail spectrum of Norcotinine, TMS derivative against reference spectrum; (b) fragments found in the simulation; (c) energy-simulation time plot (In-silico spectrum available at https://mona.fiehnlab.ucdavis.edu/spectra/display/MoNA040305)

The example showed is not a typical aromatic amide (\#566, Figure S4). The $m / z 205$ peak comes from the loss of a carbonyl group. The $\mathrm{m} / \mathrm{z} 174$ peak is missing both in the simulation and MassFrontier prediction. The $m / z 118$ peak is identified as $\mathrm{C} 7 \mathrm{H} 4 \mathrm{NO}^{+}$and $\mathrm{C} \mathrm{H} 6 \mathrm{O}^{+\bullet}$ and by their accurate masses.
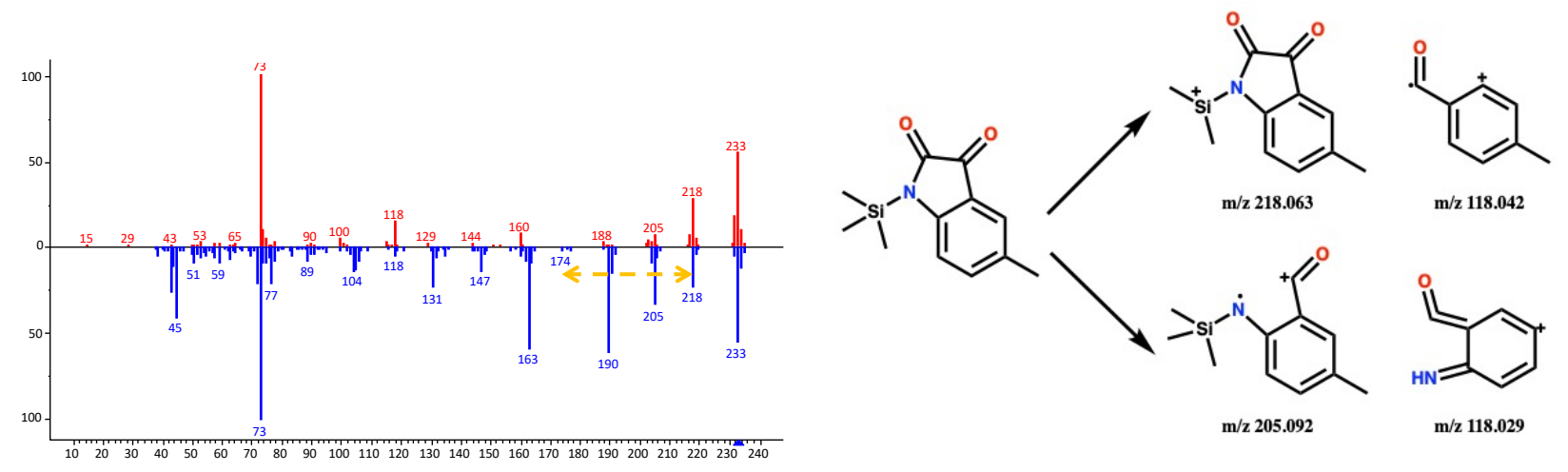

Figure S4. head-to-tail spectrum of 5-Methylisatin, TMS derivative and fragments found in simulation (In-silico spectrum available at https://mona.fiehnlab.ucdavis.edu/spectra/display/MoNA040974)

\section{Amines}


Primary amines (\#444, Figure S5) can be double or single TMS derivatives, but here we only focused on one TMS group bound to an amine group. Again, the molecular ion peak is overestimated by QCEIMS, while the other two peaks $\mathrm{m} / z 116$ and 73 match the reference quite well. There are two fragments contributing to the $m / z 116$ peak where the $\mathrm{C} 5 \mathrm{H} 14 \mathrm{NSi}^{+}$peak is the dominant one existing in 130 trajectories and the $\mathrm{C} 6 \mathrm{H} 8 \mathrm{~N}^{+}$fragment is only found in 4 trajectories. The $\mathrm{C} 6 \mathrm{H} 8 \mathrm{~N}^{+}$is an aromatic structure with many degrees of unsaturation and the trajectory shows a $\mathrm{SN}_{2}$ type rearrangement mechanism.
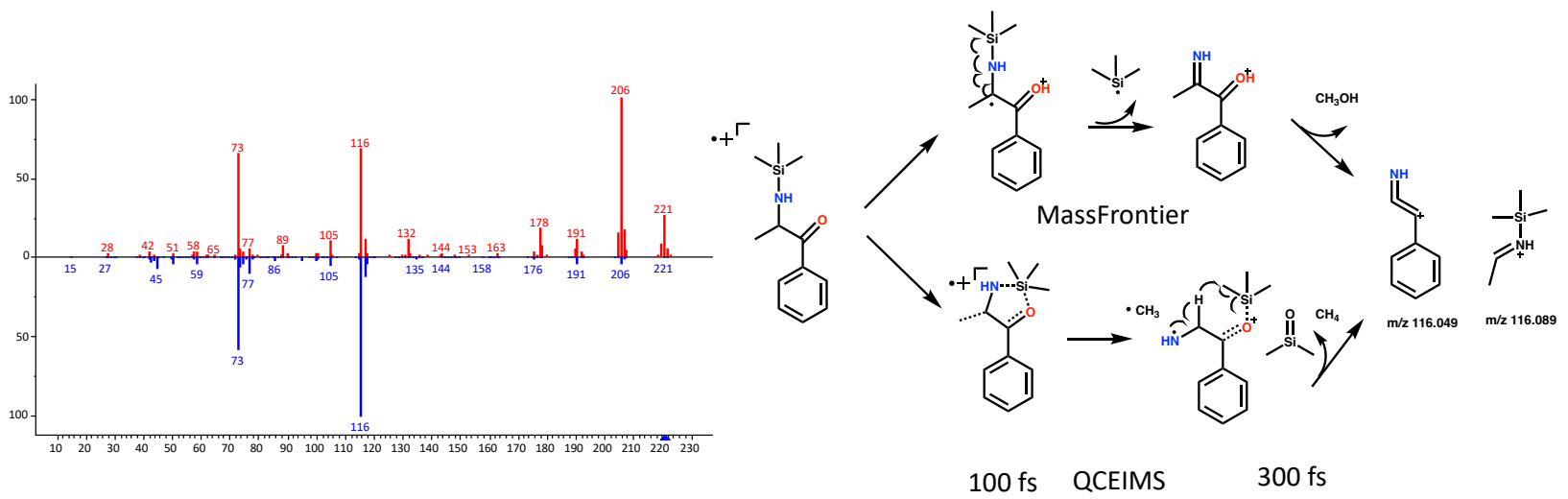

Figure S5. head-to-tail spectrum of Cathinone, TMS derivative and fragments found in simulation (In-silico spectrum available at https://mona.fiehnlab.ucdavis.edu/spectra/display/MoNA040552)

\#535(Figure S6) is an example of Aromatic amines. The Naphthalene ring made its mass spectrum simple and clean, meaning it's a good way to study the relative intensity and the reaction selectivity. The $m / z 45,59,73,215$ peaks are typical TMS derivatives peaks. The $m / z 158,157,142,141$ are resulted from the cation or radical cation stabilized by the naphthalene ring. It should be noted that the $\mathrm{m} / \mathrm{z} 100$ peak are recorded as $\mathrm{C} 8 \mathrm{H}_{4}^{+}$and $\mathrm{C} 4 \mathrm{H} 10 \mathrm{NSi}^{+}$in the simulation and lost in the MassFrontier prediction, while it is presented in the experimental reference spectrum. This reaction needs to be validated by accurate mass spectra. The abnormal structures could come from the inaccuracy of the GFN-XTB method we used, DFT level simulation should be investigated in the future.
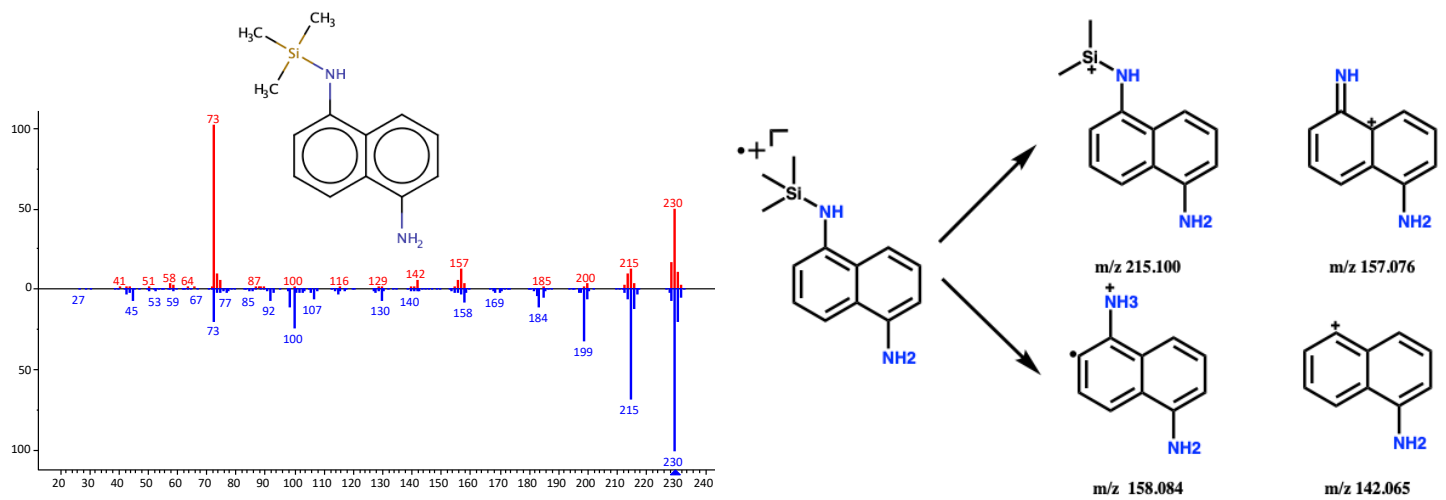

Figure S6. head-to-tail spectrum of 1,5-Diaminonaphthalene, TMS derivative and fragments found in simulation (In-silico spectrum available at https://mona.fiehnlab.ucdavis.edu/spectra/display/MoNA040622) 


\section{Thiols}

Thiol compounds exhibit many equivalents to alcohol compounds with a mass difference of 16 $\mathrm{Da}$, for example, $m / z$ 133, 106 and $91^{2}$. However, in example (\#501, Figure S7), the experimental result does not have the $[\mathrm{M}-30]+$ peak $(\mathrm{m} / z$ 196). The simulation shows an $m / z 153$ peak rather than the $\mathrm{m} / \mathrm{z} 151$ peak. The latter comes from the loss of two hydrogen atoms and an alpha cleavage. The $m / z 121$ peak results from the loss of $(\mathrm{CH} 3)_{3} \mathrm{SiS}^{\bullet}$, which is also found in other thiol compound and validated by deuterium labeling. ${ }^{4}$ The typical peak $\mathrm{m} / \mathrm{z} 106$ is also missed in the reference spectrum. The in-silico spectrum has a strong $m / z 105$ peak as $\mathrm{C}_{3} \mathrm{H} 9 \mathrm{SiS}^{+}$coming from the hydrogen atom loss from $\mathrm{m} / \mathrm{z} 106$. The $\mathrm{m} / \mathrm{z} 94$ and 93 peak also have one hydrogen difference.

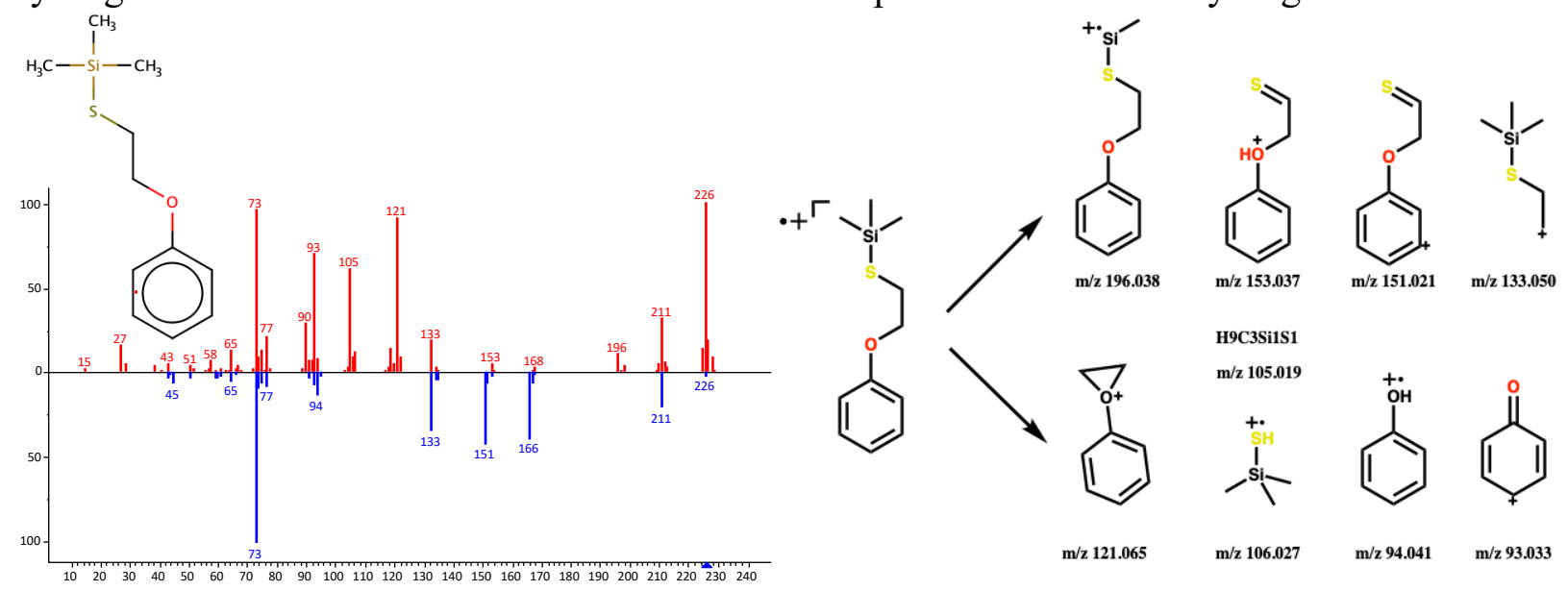

Figure S7. head-to-tail spectrum of 2-Phenoxyethanethiol, TMS derivative and fragments found in simulation

(In-silico spectrum available at https://mona.fiehnlab.ucdavis.edu/spectra/display/MoNA040782)

The aromatic thiol (\#648, Figure S8) shows a higher dot product score. The molecular ion peak is missed in the experimental reference spectrum. The $m / z 195$ has two resources C9H11OSiS ${ }^{+}(6$ trajectories) and $\mathrm{C} 8 \mathrm{H} 7 \mathrm{O} 2 \mathrm{SiS}^{+}$(32 trajectories). The $\mathrm{m} / z 136$ peak has a high intensity in the reference spectrum and the simulation generates a fragment with two five-membered-ring. The $\mathrm{m} / \mathrm{z} 89$ peak is in disagreement with the simulated and the reference spectrum. It is identified as $\mathrm{C} 4 \mathrm{H} \mathrm{O}^{+}$in the simulation and the MassFrontier software was not able to predict this reaction.
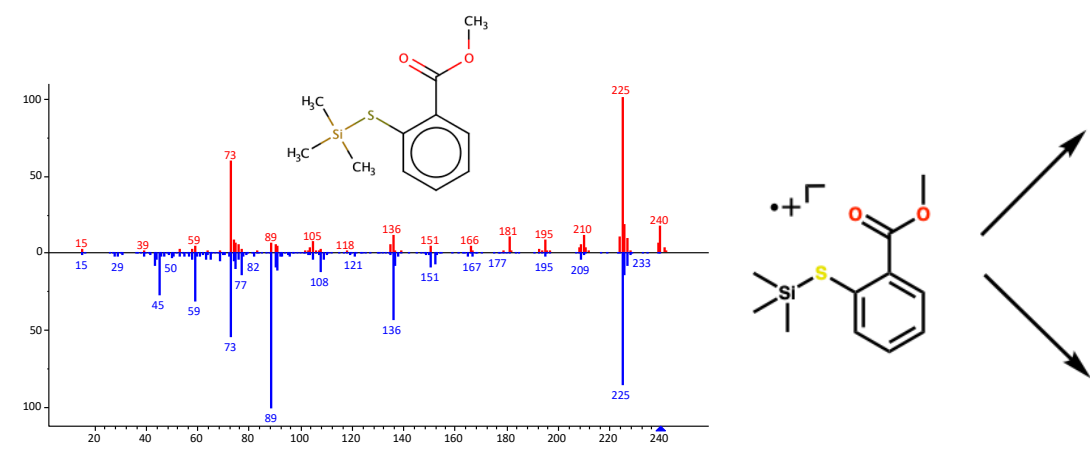

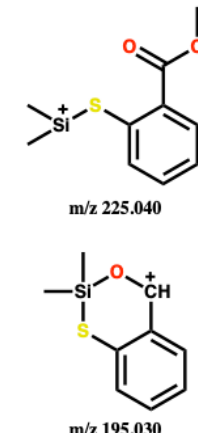

$\mathrm{m} / \mathbf{z} 195.030$<smiles></smiles>

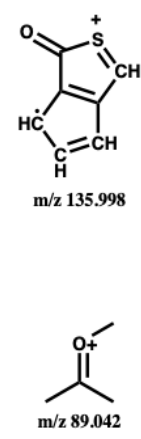


Figure S8. Head-to-tail spectrum of Methyl thiosalicylate, TMS derivative and fragments found in simulation

(In-silico spectrum available https://mona.fiehnlab.ucdavis.edu/spectra/display/MoNA040841)

\section{Accurate Mass spectra}

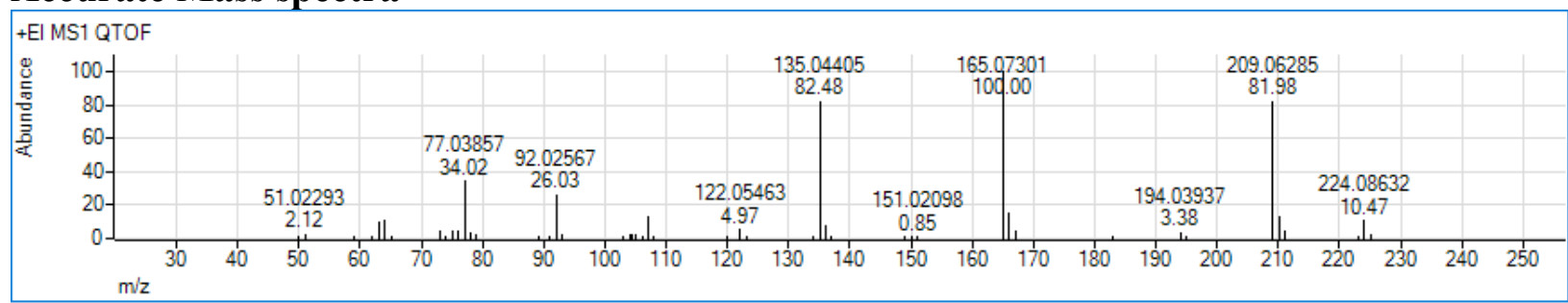

Figure S9. Accurate mass EI-MS of Trimethylsilyl 4-methoxybenzoate

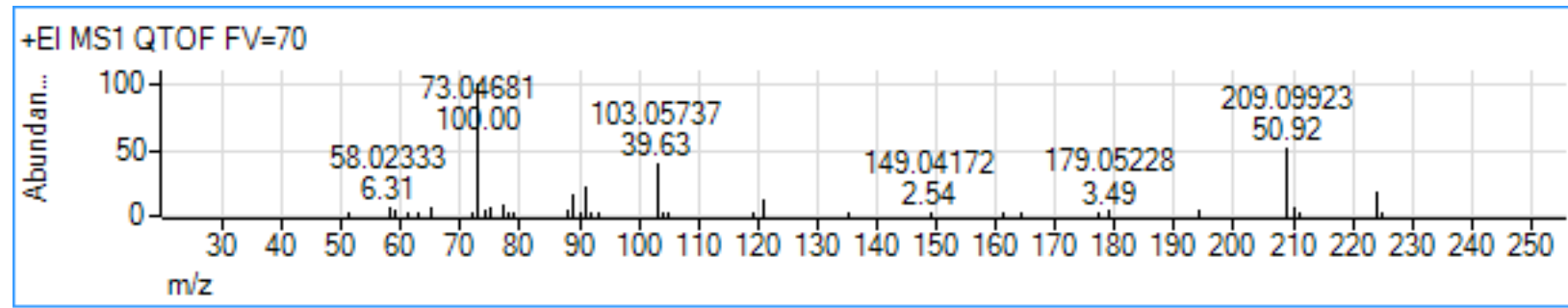

Figure S10. Accurate mass EI-MS of [2-(2-Methoxyphenyl)ethoxy](trimethyl)silane

\section{Estimate Processing time of QCEIMS TMS compounds}

Because the calculations are divided into trajectories, the program has a good ability of parallelization, while at the same time it's hard to analysis the average calculation time. Instead, we use the time of a single point calculation and assume that each trajectory has 5000 steps to estimate the running time of each molecule. Because we used two different type of CPUs, we only evaluate 464 molecules calculated on Intel Xeon E5-2699Av4 CPUs.

$$
\text { Estimate running time }=t(\text { single point }) \times 5000 \times n(\text { trajectories })
$$

Compared with our previous project5, the average calculation time increases from $1.6 \mathrm{~h}$ to $1.9 \mathrm{~h}$ (on 44 CPU threads) where better PESs and larger molecules (TMS derivatives) are simulated. Because of the better parameterization and algorithm, the QCEIMS v4.0 has a computational effort scales better than $\mathrm{O}(\mathrm{N} 2)$. Thus, the increase of simulation time can be bypassed by better parallelized property. 


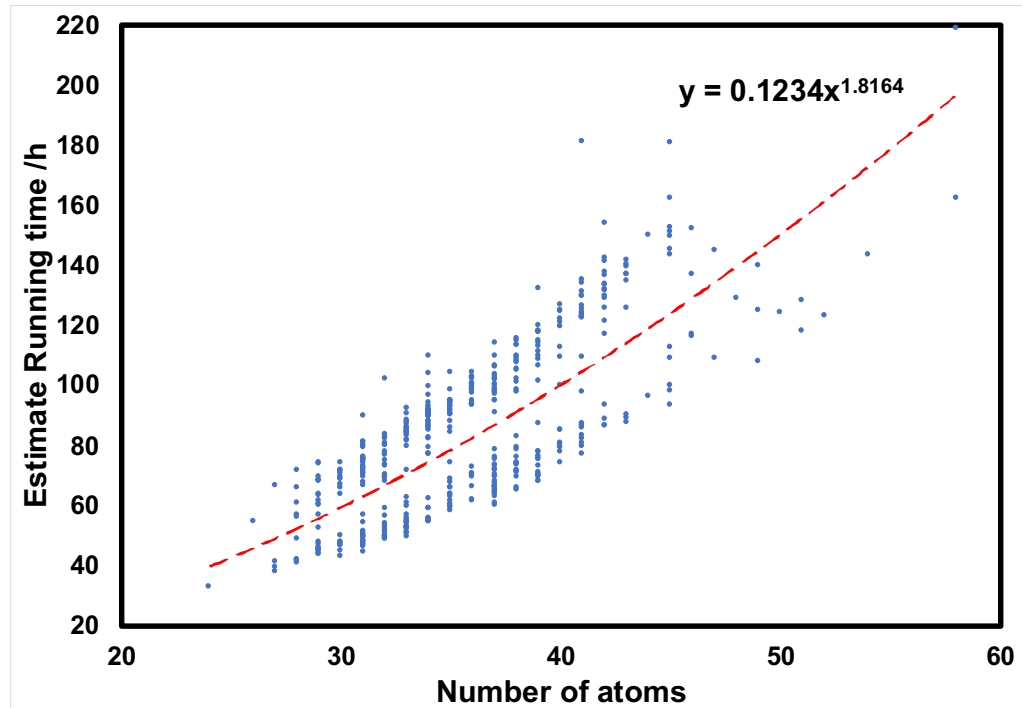

Figure S11. Processing time of 464 molecules versus the number of atoms
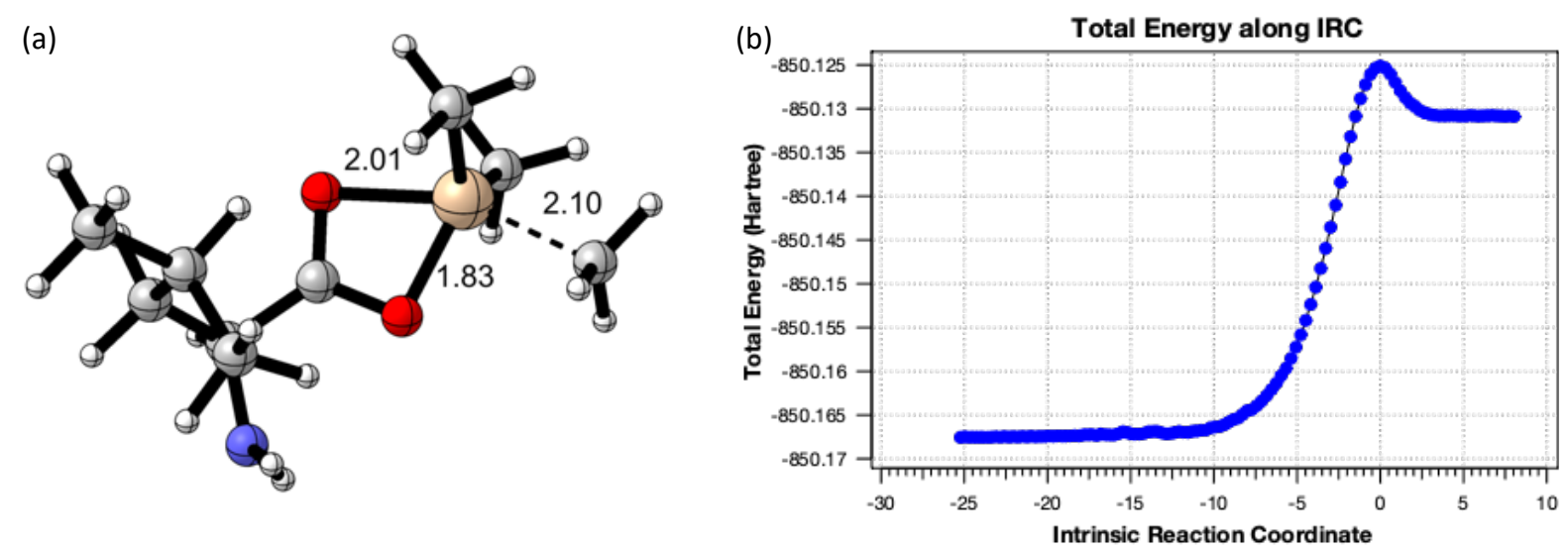

Figure S12. (a) The transition state structure of methyl group loss under $B 3 L Y P^{6-7} / 6-31 G^{8}$ level (b) The intrinsic reaction coordinate (IRC) ${ }^{9}$ calculation with Gaussian $09^{13}$ proves the transition state structure linking reactants and products. The energy barrier of this reaction is $23.18 \mathrm{kcal} / \mathrm{mol}$ under wb97xd ${ }^{10} /$ def $2-T Z V P P^{11-12}$ level.

\section{Reference}

1. Krauss, D.; Mainx, H. G.; Tauscher, B.; Bischof, P., Fragmentation of trimethylsilyl derivatives of 2-alkoxyphenols: A further violation of the 'even-electron rule'. Organic Mass Spectrometry 1985, 20 (10), 614-618.

2. Harvey, D. J.; Vouros, P., MASS SPECTROMETRIC FRAGMENTATION OF TRIMETHYLSILYL AND RELATED ALKYLSILYL DERIVATIVES. Mass Spectrometry Reviews 2020, 39 (1-2), 105-211.

3. Arafat, E. S.; Trimble, J. W.; Andersen, R. N.; Dass, C.; Desiderio, D. M., Identification of fatty acid amides in human plasma. Life Sci 1989, 45 (18), 1679-87.

4. Draffan, G. H.; Stillwell, R. N.; McCloskey, J. A., Electron impact-induced rearrangement of trimethylsilyl groups in long chain compounds. Organic Mass Spectrometry 1968, 1 (5), 669685. 
5. Wang, S.; Kind, T.; Tantillo, D. J.; Fiehn, O., Predicting in silico electron ionization mass spectra using quantum chemistry. Journal of Cheminformatics 2020, 12 (1), 63.

6. Becke, A. D., A new mixing of Hartree-Fock and local density-functional theories. The Journal of Chemical Physics 1993, 98 (2), 1372-1377.

7. Becke, A. D., Density-functional thermochemistry. III. The role of exact exchange. The Journal of Chemical Physics 1993, 98 (7), 5648-5652.

8. Ditchfield, R.; Hehre, W. J.; Pople, J. A., Self-Consistent Molecular-Orbital Methods. IX. An Extended Gaussian-Type Basis for Molecular-Orbital Studies of Organic Molecules. The Journal of Chemical Physics 1971, 54 (2), 724-728.

9. Fukui, K., The path of chemical reactions - the IRC approach. Accounts of Chemical Research 1981, 14 (12), 363-368.

10. Sengupta, A.; Raghavachari, K., Solving the Density Functional Conundrum: Elimination of Systematic Errors To Derive Accurate Reaction Enthalpies of Complex Organic Reactions. Organic Letters 2017, 19 (10), 2576-2579.

11. Schäfer, A.; Horn, H.; Ahlrichs, R., Fully optimized contracted Gaussian basis sets for atoms Li to Kr. The Journal of Chemical Physics 1992, 97 (4), 2571-2577.

12. Weigend, F.; Ahlrichs, R., Balanced basis sets of split valence, triple zeta valence and quadruple zeta valence quality for $\mathrm{H}$ to $\mathrm{Rn}$ : Design and assessment of accuracy. Phys Chem Chem Phys 2005, 7 (18), 3297-3305.

13. Gaussian 09, Revision A.02, M. J. Frisch, G. W. Trucks, H. B. Schlegel, G. E. Scuseria, M. A. Robb, J. R. Cheeseman, G. Scalmani, V. Barone, G. A. Petersson, H. Nakatsuji, X. Li, M. Caricato, A. Marenich, J. Bloino, B. G. Janesko, R. Gomperts, B. Mennucci, H. P. Hratchian, J. V. Ortiz, A. F. Izmaylov, J. L. Sonnenberg, D. Williams-Young, F. Ding, F. Lipparini, F. Egidi, J. Goings, B. Peng, A. Petrone, T. Henderson, D. Ranasinghe, V. G. Zakrzewski, J. Gao, N. Rega, G. Zheng, W. Liang, M. Hada, M. Ehara, K. Toyota, R. Fukuda, J. Hasegawa, M. Ishida, T. Nakajima, Y. Honda, O. Kitao, H. Nakai, T. Vreven, K. Throssell, J. A. Montgomery, Jr., J. E. Peralta, F. Ogliaro, M. Bearpark, J. J. Heyd, E. Brothers, K. N. Kudin, V. N. Staroverov, T. Keith, R. Kobayashi, J. Normand, K. Raghavachari, A. Rendell, J. C. Burant, S. S. Iyengar, J. Tomasi, M. Cossi, J. M. Millam, M. Klene, C. Adamo, R. Cammi, J. W. Ochterski, R. L. Martin, K. Morokuma, O. Farkas, J. B. Foresman, and D. J. Fox, Gaussian, Inc., Wallingford CT, 2016. 